\title{
CONGENITAL ATAXIA, LEARNING DISABILITIES AND AUTISM
}

Thirty two children (17 females, 15 males; mean age 12 yrs) with non-progressive congenital ataxia were examined for neuropsychiatric problems in a study at Sundsvall Hospital; Umea University Hospital; Karolinska Hospital, Stockholm; and Goteborg University, Sweden. The ataxia was a simple type in 12, an ataxic diplegia in 8 , and borderline in 12 . The majority had a mild to moderate gross motor disability and a learning disability. Fifteen of 28 with learning disability and 11 of 20 with IQs less than 50 had autistic spectrum disorder. Among 10 (31\%) children with severe learning disability, processing and integrating sensory data was a problem, auditory perception was impaired in 7 , and 5 had cerebellar pathology on neuroimaging. Sixteen $(50 \%)$ of the 32 patients with ataxia had symptoms of autistic spectrum disorder. Eight $(25 \%)$ had attention deficit hyperactivity disorder, associated with autistic traits in 3. Learning disability, often combined with ADHD, and autistic spectrum disorder were strongly associated, as was simple and borderline ataxia, whereas ataxic diplegia and neuropsychiatric disorders formed a weaker link. Neuroimaging in all 12 with simple ataxia revealed abnormalities in 7 , including cerebellar hypolasia, vermal hypolasia, corpus callosum malformation, and Dandy-Walker malformation. In patients with ataxic diplegia, neuroimaging showed gliosis and atrophy in the thalami, and supratentorial atrophy following perinatal hemorrhage. None of the borderline ataxic cases showed cerebellar pathology on MRI or CT. Prenatal malformations and chromosomal abnormalities and syndromes were the most frequent causes of simple and borderline congenital ataxias and associated learning disabilities and autism. The origin of the ataxic diplegias was more often a perinatal hypoxic ischemic encephalopathy, only one having a chromosome anomaly. Children with congenital ataxia should receive an MRI and chromosomal and metabolic investigations. They should be followed up and evaluated for learning disability (including ADHD) and autistic spectrum disorders. (Ahsgren I, Baldwin I, Goetzinger-Falk $\mathrm{C}$ et al. Ataxia, autism, and the cerebellum: a clinical study of 32 individuals with congenital ataxia. Dev Med Child Neurol March 2005;47:193-198). (Respond: Ingegerd Ahsgren MD, Department of Child and Adolescent Neurology and Habilitation, Norrliden, Sundsvall Hospital, SE 85186 Sundsvall, Sweden).

COMMENT. The authors highlight the common association of congenital nonprogressive forms of ataxia with learning disability, ADHD, and autistic spectrum disorder. The causes are heterogeneous, but prenatal factors, including chromosomal malformations, are most common with the simple ataxias, whereas perinatal hypoxic ischemic pathology is the usual cause of ataxic diplegia. Children with congenital ataxia are at risk for learning disability, ADHD, and autistic disorders.

Hypoplasia of the cerebellar vermis and cognitive deficits in children with acute lymphoblastic leukemia treated with cranial radiation are reported (Ciesielski KT et al. Arch Neurol 1994;51:985-993; Ped Neur Briefs Nov 1994). Patients treated before 5 years of age are more susceptible to cognitive deficits than older children. Visual-spatial-motor coordination and memory were most involved, but behavioral and autistic symptoms were not reported. Hypoplasia of the cerebellar vermis is described in association with autism and in males with fragile X syndrome, frequently complicated by autism (Courchesne $\mathrm{E}$ et al. $\mathbf{N}$ Engl J Med 1988;318:1349; Ped Neur Briefs Feb 1991). The cerebellum has been linked with neurocognitive and neurobehavioral symptoms for more than a decade. 
Fragile X-associated tremor/ataxia syndrome (FXTAS) is described in 2 sisters (BerryKravis E et al. Ann Neurol Jan 2005;57:144-147). The sisters were premutation carriers who developed postural tremor at 75 and 83 years of age, progressing to further disabilities with ataxia in the younger one, whose MRI showed increased signal in the cerebellar white matter and middle peduncle, and in subcortical white matter. The older patient had 2 children with FXS and cognitive disability. The father of the 2 sisters and his brother had parkinsonian tremor. Both sisters had nonrandom X-inactivation that dictated symptom severity. Symptoms of FXTAS in the women were milder than those in men in the family. This report demonstrates the association of cerebellar pathology and ataxia with a mental retardation genetic developmental syndrome.

\section{ATTENTION DEFICIT DISORDERS}

\section{EEG THETA ACTIVITY, COGNITIVE DISORDERS AND ADHD}

The relation between resting EEG theta activity and poor performance in attentiondemanding cognitive tasks in 46 unmedicated children/adolescents with attention deficit hyperactivity (ADHD) was studied at Westmead Hospital, University of Sydney, NSW, Australia; and Heinrich-Heine University of Dusseldorf, Germany. Accuracy and reaction time during an auditory oddball signal detection test and a visual continuous performance test were recorded. EEG power spectral analyses revealed an increase in frontal (primarily left frontal) theta activity for ADHD subjects compared to controls $(\mathrm{F}=4,18, \mathrm{p}<0.05)$. Compared with control subjects, the ADHD group had significantly delayed reaction times and decreased accuracy in both cognitive tasks. In the ADHD group, correlation analyses revealed a significant relation between accuracy on the oddball task and left frontal theta, and in controls, a significant relationship between reaction time in the continuous performance test and right posterior theta. Resting theta is interpreted as an index of inattention, and spatial neurophysiologic deficits in ADHD may be related to disturbed signal detection and inattention. (Hermens DF, Soei EXC, Clarke SD et al. Resting EEG theta activity predicts cognitive performance in attention-deficit hyperactivity disorder. Pediatr Neurol April 2005;32:248-256). (Respond: Dr Hermens, The Brain Dynamics Centre, Acacia House, Westmead Hospital, Hawkesbury Road, Westmead, NSW, 2145, Australia).

COMMENT. This report confirms the finding of increased theta activity in patients with ADHD, and suggests that the evidence may be predictive of errors in signal detection and attention. The authors comment that the localization of the increase in theta to the left frontal brain is consistent with reports of neuroimaging data showing a reduction in left frontal cerebral white matter. These data also refer to reduction in volume of right frontal gray matter (Mostofsky SH et al. Smaller prefrontal and premotor volumes in boys with attention deficit/hyperactivity disorder. Biol Psychiatry 2002;52:785-794). Previous MRI studies have shown a decreased volume of the prefrontal cortex, caudate nucleus, and globus pallidus on the right side, pointing to a dysfunction of right-sided prefrontal-striatal systems in ADHD children (Castellanos FX et al. Arch Gen Psychiatry 1996;53:607-616; Ped Neur Briefs Aug 1996). 